\title{
Successful management of placenta percreta by cesarean hysterectomy with transverse uterine fundal incision
}

\section{Shinya Matsuzaki*, Kiyoshi Yoshino, Etsuko Kajimoto, Yusuke Tanaka, Kazuya Mimura, Takeshi Kanagawa, Tadashi Kimura}

Department of Obstetrics \& Gynecology, Osaka University Graduate School of Medicine, Osaka, Japan

Received: 26 March 2014

Accepted: 14 April 2014

\author{
*Correspondence: \\ Dr. Shinya Matsuzaki, \\ E-mail: zacky@gyne.med.osaka-u.ac.jp
}

(C) 2014 Matsuzaki S et al. This is an open-access article distributed under the terms of the Creative Commons Attribution Non-Commercial License, which permits unrestricted non-commercial use, distribution, and reproduction in any medium, provided the original work is properly cited.

\begin{abstract}
Placenta accreta presents one of the highest risks to pregnancy, and its more severe variant, placenta percreta, is particularly risky. The incidence of both conditions is increasing. Placenta percreta requires a cesarean hysterectomy for management, but the challenges associated with this surgery often result in severe obstetric hemorrhaging and high rates of maternal morbidity. Several recent obstetric studies have reported on the usefulness of the transverse uterine fundal incision for the management of placenta accreta and its variants. However, these reports included only a few cases of placenta percreta. Here we present a case of placenta percreta covering the anterior uterine wall that was successfully managed using a transverse fundal incision, which avoided incising the placenta at delivery and thus reduced maternal blood loss. After delivery, the patient underwent a total abdominal hysterectomy without the need for a blood transfusion. We conclude that a transverse uterine fundal incision can be very useful for the management of placenta percreta of the anterior uterine wall.
\end{abstract}

Keywords: Placenta previa, Placenta accreta, Placenta percreta, Transverse uterine fundal incision, Cesarean hysterectomy

\section{INTRODUCTION}

Placenta previa is an obstetric complication wherein the placenta is inserted in the lower uterine segment. Placenta percreta, a more severe variant of placenta accreta, in which the placenta abnormally penetrates through the myometrium to the uterine serosa, is the most serious complication of placenta previa ${ }^{1}$ and is associated with heavy obstetrical hemorrhaging. ${ }^{2}$ A well-known risk factor for placenta percreta is a history of one or more previous cesarean deliveries. ${ }^{3}$ Management of placenta percreta requires a cesarean delivery and total abdominal hysterectomy, but the high risk of significant blood loss makes this surgery very difficult. Moreover, the optimal surgical approach to use in these cases has not been established. We encountered a case of placenta percreta covering the entire anterior uterine wall, which is the rarest and most complicated form of placenta percreta.
Here we report the successful use of a transverse fundal incision to avoid incising the placenta, followed by a total abdominal hysterectomy that did not require a blood transfusion.

\section{CASE REPORT}

A 41-year-old female (gravida 7, para 3) was referred to our hospital from a private clinic at 28 weeks of gestation because of placenta previa. The patient had a history of three cesarean deliveries, and in her current pregnancy, the placenta was located deep into the anterior uterine wall and totally embedded within the lower uterine segment. We performed ultrasonography and magnetic resonance imaging and diagnosed her with placenta percreta (Figure 1a). Profuse bleeding occurred during the 29th week of gestation, at which time the patient was 
admitted to the hospital. Fetal growth was determined to be appropriate for the gestational age.

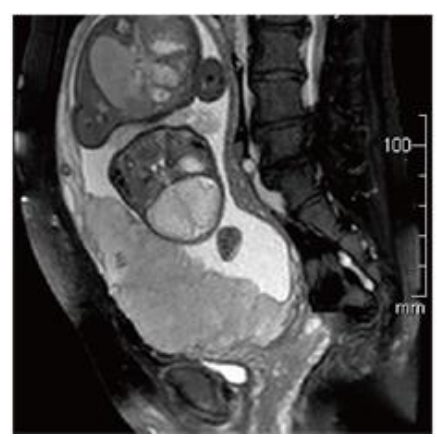

Figure 1a: Pelvic magnetic resonance imaging suggested placenta percreta.

The patient was considered at risk for severe obstetrical complications due to placenta percreta; therefore, a cesarean delivery followed by hysterectomy was planned for 34 weeks of gestation to avoid emergent surgery. To address the possibility of placenta percreta, a multidisciplinary team, including specialists from obstetrics, perinatology, gynecology, urology, and interventional radiology, was assembled, and crossmatched blood was available at all times during her hospitalization. Antenatal corticosteroids for fetal lung maturation were not administered because the scheduled delivery was planned for after 34 weeks of gestation. We planned to use a transverse fundal incision to avoid incising the placenta, followed by hysterectomy.

On the scheduled day of surgery, a cesarean delivery was performed. Laparotomy showed that the placenta was located in the lower uterine segment, and large blood vessels were observed in uterine lower segment (Figure 1b). From these findings, we suspected placenta percreta. After elevating the uterus outside the abdominal wall, an ultrasonographically guided transverse incision was performed at the uterine fundus to avoid rupturing the membrane or producing a bulge on the fetal membrane (Figure 2a and 2b). The incision resulted in minimal bleeding, and a healthy male with a birth weight of 2644 $\mathrm{g}$ was successfully delivered.

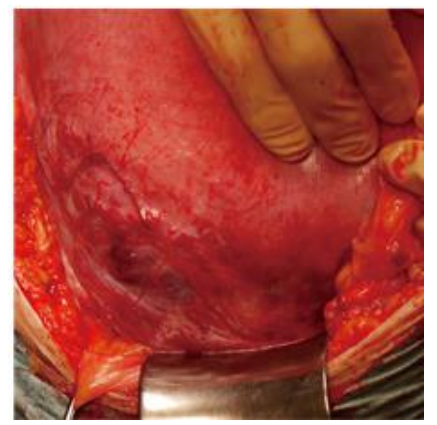

Figure 1b: After laparotomy, large blood vessels and part of the placenta could be seen through the anterior uterine wall.

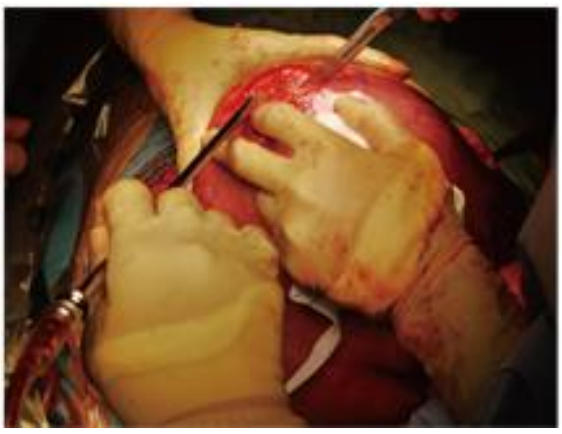

Figure 2a: A transverse incision into the uterine fundus was performed, taking care to avoid rupture of the fetal membranes. Minimal bleeding was observed from the incision.

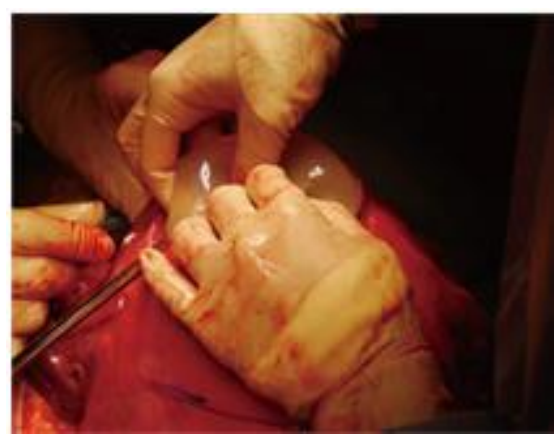

Figure 2b: The unruptured fetal membranes can be seen bulging through the transverse fundal incision.

We then performed a hysterectomy. Abnormal adherence was observed between the anterior uterine wall and the bladder, making it difficult to separate the bladder from the uterus, and approximately $1000 \mathrm{~mL}$ of bleeding was observed. However, because the transverse uterine fundal incision avoided iatrogenic placental separation, bleeding was not observed from the placenta. The hysterectomy was completed without complications, and the abdomen was closed. The total estimated blood loss was approximately $1500 \mathrm{~mL}$. After surgery, the patient's hemoglobin concentration was $9.4 \mathrm{~g} / \mathrm{dL}$, and a blood transfusion was not required. The patient had an uncomplicated postoperative course and was discharged 14 days later in good condition.

\section{DISCUSSION}

Several studies have reported an association between the risk for placenta accreta and the number of prior cesarean deliveries. ${ }^{3}$ In addition to multiple previous cesarean deliveries, placenta previa is the most serious risk factor for placenta accreta. ${ }^{1}$ Because the rate of cesarean deliveries continues to increase, it is expected that the prevalence of placenta percreta will also continue to increase.

Placenta percreta is one of the most significant complications of pregnancy because of the risk of heavy obstetrical hemorrhage. A recent study reported that the 
average blood loss during surgery for placenta percreta is $4,800 \mathrm{~mL}$, which increases the difficulty of the operative technique. ${ }^{2}$ Numerous studies have reported the benefits of various methods for the management of placenta percreta at delivery, including conservative management, uterine artery embolization, balloon occlusion, the use of uterine compression sutures for postpartum haemorrhage, and planned hysterectomy. ${ }^{2,4-7}$ However, these reports have also suggested that the best methods of delivery and management remain controversial and should be determined on a case-by-case basis. In the present case, the patient was diagnosed with placenta percreta and desired no further children; therefore, we planned a cesarean hysterectomy. While bleeding may be reduced by the use of a balloon occlusion technique, its safety has not yet been established..$^{5}$ Therefore, in our case, balloon occlusion was not used.

For cases such as ours, in which the placenta percreta covers the entire anterior uterine wall, the optimal cesarean delivery method has not been established. ${ }^{8}$ In our case, vertical incision at a distance from the placenta would have been difficult because the placenta broadly involved the anterior uterine wall. Recent studies have reported that the use of a transverse uterine fundal incision for the management of placenta accreta can effectively avoid cutting into the placenta and consequently reduce fetal and maternal blood loss. ${ }^{9,10}$ This technique presented distinct advantages over a transverse lower uterine segment incision or a vertical uterine incision. It is important to note, however, that one drawback of this procedure is the limited data regarding the effect on subsequent pregnancies. Therefore, we are currently not able to recommend this procedure for patients who desire subsequent pregnancies.

Kotsuji F et al. reported 19 cases, and Nishida et al. reported 8 cases of transverse fundal incision for placenta accreta. ${ }^{9,10}$ However, in these reports, the total number of cases involving placenta percreta seemed to be only two. Our case is a relatively rare report of the successful management of placenta percreta with a transverse fundal incision and subsequent total abdominal hysterectomy without blood transfusion. Further reports are needed to investigate the usefulness of this surgical method for placenta percreta covering the anterior wall of the uterus.

In summary, we successfully managed a case of placenta percreta through transverse fundal incision and planned hysterectomy and consequently, we were able to minimize blood loss. Therefore, we propose that transverse uterine fundal incision and planned hysterectomy should be considered for patients with suspected placenta accreta and its variants who do not desire subsequent pregnancies.

\section{ACKNOWLEDGMENTS}

We thank A. Yagi and K. Sakiyama for secretarial assistance.

\section{Funding: No funding sources \\ Conflict of interest: None declared \\ Ethical approval: Not required}

\section{REFERENCES}

1. Belfort MA. Placenta accreta. Am J Obstet Gynecol. 2010;203:430-9.

2. Clausen C, Lonn L, Langhoff-Roos J. Management of placenta percreta: a review of published cases. Acta Obstet Gynecol Scand. 2014;93:138-43.

3. Silver RM, Landon MB, Rouse DJ, Leveno KJ, Spong CY, Thom EA et al. Maternal morbidity associated with multiple repeat cesarean deliveries. Obstet Gynecol. 2006;107:1226-32.

4. Sumigama S, Itakura A, Ota T, Okada M, Kotani T, Hayakawa $\mathrm{H}$ et al. Placenta previa increta/percreta in Japan: a retrospective study of ultrasound findings, management and clinical course. J Obstet Gynaecol Res. 2007;33:606-11.

5. Clausen C, Stensballe J, Albrechtsen CK, Hansen MA, Lonn L, Langhoff-Roos J. Balloon occlusion of the internal iliac arteries in the multidisciplinary management of placenta percreta. Acta Obstet Gynecol Scand. 2013;92:386-91.

6. Wong VV, Burke G. Planned conservative management of placenta percreta. J Obstet Gynaecol. 2012;32:447-52.

7. Canonico S, Arduini M, Epicoco G, Luzi G, Arena $S$, Clerici $G$ et al. Placenta previa percreta: a case report of successful management via conservative surgery. Case Rep Obstet Gynecol. 2013;2013:702067.

8. Ward CR. Avoiding an incision through the anterior previa at cesarean delivery. Obstet Gynecol. 2003;102:552-4.

9. Kotsuji F, Nishijima K, Kurokawa T, Yoshida Y, Sekiya T, Banzai $M$ et al. Transverse uterine fundal incision for placenta praevia with accreta, involving the entire anterior uterine wall: a case series. BJOG. 2013;120:1144-9.

10. Nishida R, Yamada $T$, Akaishi R, Kojima $T$, Ishikawa $\mathrm{S}$, Takeda $\mathrm{M}$ et al. Usefulness of transverse fundal incision method of cesarean section for women with placentas widely covering the entire anterior uterine wall. J Obstet Gynaecol Res. 2013;39:91-5.

DOI: 10.5455/2320-1770.ijrcog20140633

Cite this article as: Matsuzaki S, Yoshino K, Kajimoto E, Tanaka Y, Mimura K, Kanagawa T, Kimura T. Successful management of placenta percreta by cesarean hysterectomy with transverse uterine fundal incision. Int J Reprod Contracept Obstet Gynecol 2014;3:447-9. 